\title{
Case Report \\ Hepatitis E Infection in a Renal Transplant Recipient
}

\author{
Diana Vassallo, ${ }^{1}$ Mir Mubariz Husain, ${ }^{2}$ Shaun Greer, ${ }^{3}$ Stephen McGrath, ${ }^{4}$ \\ Samreen Ijaz, ${ }^{5}$ and Durga Kanigicherla ${ }^{1}$ \\ ${ }^{1}$ Department of Renal Medicine, Central Manchester NHS Foundation Trust, Manchester Royal Infirmary, Oxford Road, \\ Manchester M13 9WL, UK \\ ${ }^{2}$ Department of Clinical Virology, Central Manchester NHS Foundation Trust, Manchester M13 9WL, UK \\ ${ }^{3}$ Department of Hepatology, Central Manchester NHS Foundation Trust, Manchester M13 9WL, UK \\ ${ }^{4}$ Department of Pathology, Central Manchester NHS Foundation Trust, Manchester M13 9WL, UK \\ ${ }^{5}$ Blood Borne Virus Unit, MS-Colindale, Public Health England, London NW9 5EQ, UK \\ Correspondence should be addressed to Diana Vassallo; vassallodi@gmail.com
}

Received 18 May 2014; Accepted 29 July 2014; Published 11 September 2014

Academic Editor: Raoul Bergner

Copyright (C) 2014 Diana Vassallo et al. This is an open access article distributed under the Creative Commons Attribution License, which permits unrestricted use, distribution, and reproduction in any medium, provided the original work is properly cited.

\begin{abstract}
An asymptomatic 35-year-old renal transplant recipient was noted to have deranged liver function tests. Liver biopsy revealed a portal inflammatory process with mild lobular activity and portal fibrous expansion, consistent with a virally mediated process. An extensive viral screen confirmed infection with Hepatitis E virus genotype 3 (HEV-3). There is increased awareness about locally acquired Hepatitis E virus (HEV) infection in the transplant population in the UK. The important implications of this infection are becoming more apparent as progression to liver cirrhosis can occur. However, the incidence, natural history, and treatment of HEV infection in the transplant population are not well established. This report illustrates a case of delayed spontaneous clearance of the HEV infection.
\end{abstract}

\section{Introduction}

In recent years, there has been increased interest in $\mathrm{HEV}$ infection as emerging data has revealed that chronic infection can lead to progressive liver disease in immunosuppressed patients including various transplant cohorts such as heart and lung transplant recipients $[1,2]$. Chronic Hepatitis E should be considered in the differential diagnosis of elevated liver enzymes in transplant recipients. The frequency and course of these infections are however not well defined. The following case report highlights the challenges involved in diagnosis and treatment of this condition in a kidney transplant recipient and illustrates a case of delayed spontaneous clearance of the infection.

\section{Case Report}

A 35-year-old gentleman received a deceased donor renal transplant in 2006 following end-stage renal failure secondary to focal sclerosing glomerulosclerosis (FSGS). Basiliximab was used as induction immunosuppression followed by maintenance therapy with ciclosporin and prednisolone. Over the subsequent years, there was persistent moderate graft dysfunction with serum creatinine of $200 \mu \mathrm{mol} / \mathrm{L}$. Graft biopsies revealed chronic interstitial fibrosis and tubular atrophy. An episode of T-cell mediated rejection in 2009 (Banff 1A on biopsy) was treated with pulsed methylprednisolone and mycophenolate mofetil (MMF) was commenced. Graft function continued to deteriorate and in April 2010 ciclosporin was stopped. His immunosuppression at this stage consisted of MMF $1 \mathrm{~g}$ bid and prednisolone $5 \mathrm{mg}$ daily.

In April 2011 he was referred to the transplant clinic. As part of the work-up towards listing for subsequent kidney transplantation, deranged liver function tests (LFTs) were noted. A sharp rise in alanine transaminase (ALT) had occurred around April 2010 (Figure 1). There was no past history of jaundice, nor any change to his medication around this time. There had been no change in alcohol intake, nor any significant change in weight. He had gone on holiday to Tenerife in November 2009 and no illnesses had been reported during this trip. Clinical examination revealed a mildly obese gentleman with abdominal striae but there were no stigmata of chronic liver disease. 


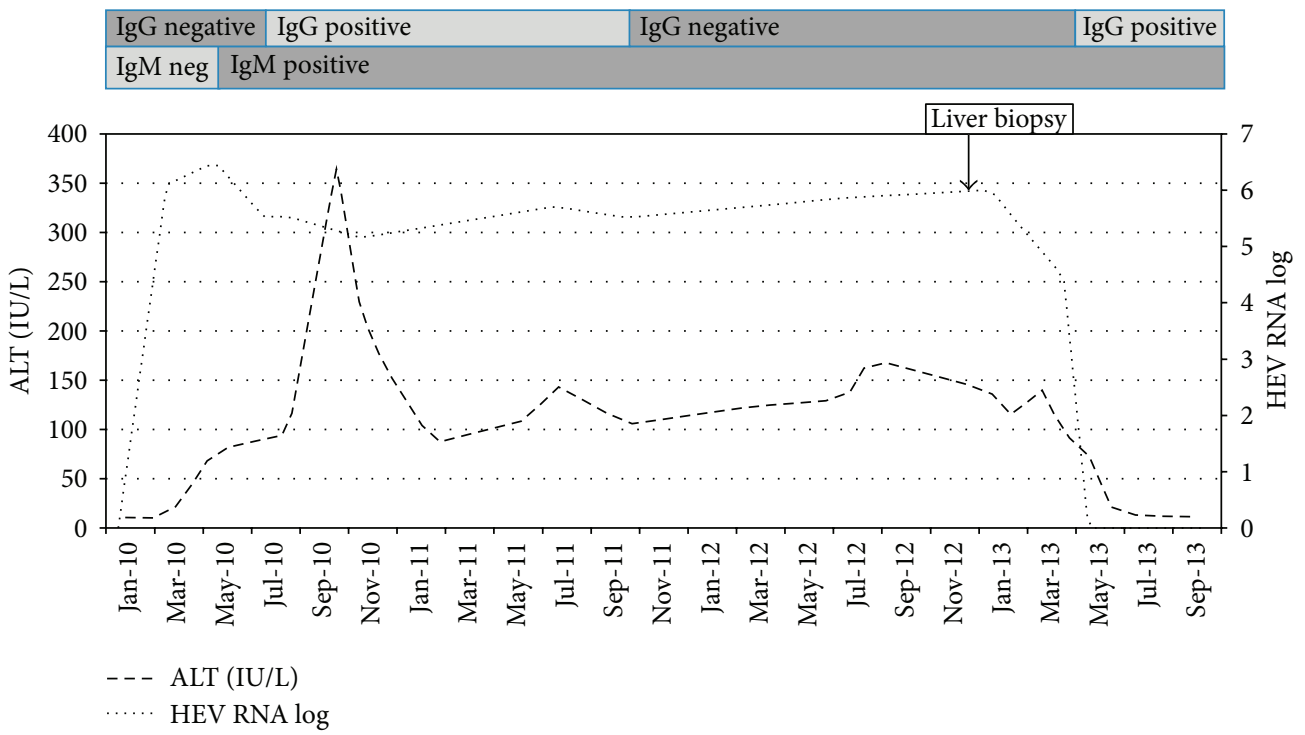

FIGURE 1: Evolution of alanine transaminase (ALT) levels, Hepatitis E virus (HEV) RNA levels, and HEV serology.

Ultrasound of the liver revealed a bright echotexture in keeping with fatty infiltration. Routine liver and immunology screen did not reveal any abnormality, including Hepatitis B, Hepatitis C, human immunodeficiency virus (HIV), cytomegalovirus (CMV), and Epstein-Barr viral (EBV) titres. The presumed diagnosis was steatohepatitis, although this did not explain the acute rise in LFTs. Further evaluation was not possible at this stage due to missed hepatology appointments, until a liver biopsy was performed in January 2013.

There was evidence of relatively mild fibrosis involving the portal tracts only, some of which appeared expanded and larger than usual. Features of more advanced fibrosis, such as bridging fibrosis linking adjacent portal structures and nodule formation, were not identified (Figure 2). The appearance was consistent with that of a virally mediated process. A more extensive viral screen was performed and serology was consistent with active Hepatitis E virus (HEV) infection. Detection of HEV antibody was carried out using the Wantai IgM and IgG detection assays (Fortress Diagnostics, Northern Ireland). The assays were run in accordance with the manufacturer's instructions. Subsequently, nucleic acid was extracted from serum using the Magna Pure 96 platform (Roche diagnostics Ltd, UK). HEV ribonucleic acid (RNA) detection and quantification were then undertaken via a real time assay [3]. This confirmed HEV genotype 3 (HEV-3) infection. HEV RNA detection and quantification were undertaken using qPCR but genotyping was done on a separate assay. This is based on PCR amplification and Sanger sequencing of a 300 base pair (bp) region across the open reading frame (ORF) 2 as published previously [4]. Retrospective serological and virological assays were performed on blood samples dating back to 2009.

The patient's immunosuppressive regime was not altered during this time. In April 2013 LFTs normalised spontaneously and the HEV RNA levels became negative (Figure 1). This remains negative to date. The patient started haemodialysis in January 2013 following graft failure and is now on the national waiting list for kidney transplantation.

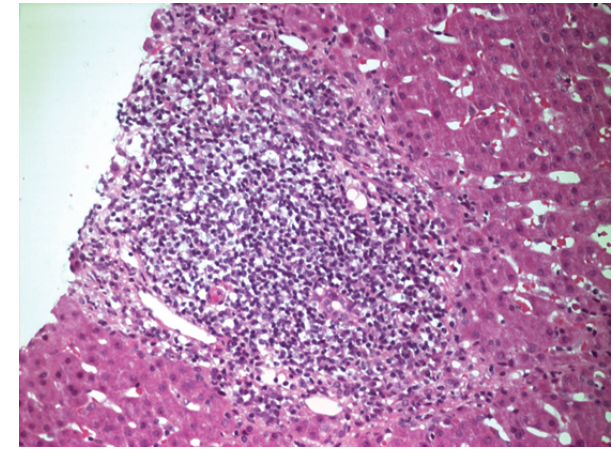

FIGURE 2: Liver biopsy showing a portal tract containing a dense, predominantly lymphocytic infiltrate. There is minimal inflammation affecting the peripheral interface region of the portal tract. The portal vein, hepatic artery, and several biliary structures can be seen within the infiltrate (haematoxylin and eosin stain, magnification $\times 20)$.

\section{Discussion}

HEV infection in transplant recipients has gained more recognition in recent years. Emerging knowledge about this infection has raised concerns specific to this patient cohort [5]. In immunocompetent individuals, HEV generally manifests itself as a self-limiting acute viral hepatitis, with the exception of a fulminant presentation in pregnant women and in patients with chronic liver disease, although this has only been described in developing countries, mainly due to waterborne HEV genotype 1 [6]. However, it is known that HEV can lead to chronic hepatitis in approximately $60 \%$ of infected solid organ transplant recipients and $14.3 \%$ of these develop liver cirrhosis $[5,7,8]$.

$\mathrm{HEV}$ is now recognised as an endemic or autochthonous infection in Europe and is no longer considered an imported 
infection from hyperendemic developing countries [9]. HEV3 is distributed worldwide and is a porcine zoonosis associated with consumption of pork products $[9,10]$. While on holiday in Tenerife, our patient consumed several pork-based meals. The incubation period in immunocompetent individuals is between 2 and 6 weeks [8] although this has not been characterised in transplant patients. It is not possible for us to distinguish between the Spanish and UK HEV-3 with confidence. However, given that in our report HEV RNA was detected more than 4 months following the patient's last holiday, it appears that likely the infection was acquired locally in the UK.

In the last few years, awareness of the HEV burden in developed countries, especially in the immunosuppressed cohort, has increased [11]. Most of the reported cases have been diagnosed in Southern France [12] where the incidence of HEV among liver, kidney, or simultaneous kidneypancreas transplantation has been quoted as 3.2 cases/100 person-years [8]. In the UK, there is increased awareness of HEV infection amongst the general population and a public health issue was recognised in 2005 due to a significant number of infections in the general population [13]. A HEV-IgG seroprevalence rate of $16 \%$ has been detected in blood donors in Southwest England [14]. However, the incidence of HEV infection in transplant recipients in the UK is unknown.

Clinical suspicion is essential to enable a timely diagnosis. Transplant recipients are on a multitude of potentially hepatotoxic medication. In addition, HEV infections can be relatively asymptomatic with modest elevations in transaminases even in healthy individuals. Serological assays show a range of sensitivities and specificities and HEV serology may be negative in immunocompromised individuals [15]. Our patient became HEV-IgG positive around the time of detectable HEV RNA load; however, the IgG response was quite weak, just harbouring above the assay cut-off. HEV-IgG levels become undetectable in September, 2011, and detectable again in May 2013. However this is probably not reseroconversion but reflects fluctuation of a low IgG signal around the assay cutoff. The diagnosis of HEV can only be confirmed by performing HEV RNA detection studies [16]. HEV RNA testing is increasingly becoming more accessible and some commercial assays are now available. These diagnostic challenges raise the possibility that the burden of this infection in transplant recipients is underestimated.

Around $34 \%$ of immunosuppressed patients infected with HEV spontaneously clear the virus within 6 months, without any change in underlying immunosuppression or use of antiviral agents [7]. In our case, the patient cleared the virus spontaneously at approximately 36 months of infection. Delayed spontaneous clearance was reported in one other case in the literature, where the viral clearance occurred after 42.1 months [5]. It is not clear what triggered viral clearance in our patient as there was no change to his immunosuppression or in his clinical condition, at and a few months preceding the time of viral clearance, apart from the commencement of haemodialysis in January 2013.

In conclusion, HEV infection can be acquired locally and should be considered as a differential diagnosis of unexplained deranged liver function tests in transplant recipients.
Screening for HEV by PCR should be routinely done in such cases. There is a need for more data regarding incidence and outcomes of HEV infection in transplant recipients in the UK. Factors that trigger spontaneous clearance of these infections are poorly understood. Increased awareness of locally acquired infections will help optimise management by enabling early diagnosis and contributing to knowledge about effective treatment and possible preventative measures.

\section{Conflict of Interests}

The authors declare that there is no conflict of interests regarding the publication of this paper.

\section{Acknowledgment}

The authors would like to thank the Department of Clinical Virology at the Central Manchester University Hospitals NHS Foundation Trust.

\section{References}

[1] S. Pischke, P. Stiefel, B. Franz et al., "Chronic hepatitis E in heart transplant recipients," The American Journal of Transplantation, vol. 12, no. 11, pp. 3128-3133, 2012.

[2] S. Pischke, M. Greer, S. Hardtke et al., "Course and treatment of chronic hepatitis E virus infection in lung transplant recipients," Transplant Infectious Disease, vol. 16, no. 2, pp. 333-339, 2014.

[3] J. A. Garson, R. B. Ferns, P. R. Grant et al., "Minor groove binder modification of widely used TaqMan probe for hepatitis $\mathrm{E}$ virus reduces risk of false negative real-time PCR results," Journal of Virological Methods, vol. 186, no. 1-2, pp. 157-160, 2012.

[4] S. Ijaz, E. Arnold, M. Banks et al., "Non-travel-associated hepatitis E in England and Wales: demographic, clinical, and molecular epidemiological characteristics," Journal of Infectious Diseases, vol. 192, no. 7, pp. 1166-1172, 2005.

[5] N. Kamar, H. R. Dalton, F. Abravanel, and J. Izopet, "Hepatitis E virus infection," Clinical Microbiology Reviews, vol. 27, no. 1, pp. 116-138, 2014.

[6] E. H. Teshale, D. J. Hu, and S. D. Holmberg, "The two faces of hepatitis E virus," Clinical Infectious Diseases, vol. 51, no. 3, pp. 328-334, 2010.

[7] N. Kamar, C. Garrouste, E. B. Haagsma et al., "Factors associated with chronic hepatitis in patients with hepatitis E virus infection who have received solid organ transplants," Gastroenterology, vol. 140, no. 5, pp. 1481-1489, 2011.

[8] N. Kamar, F. Legrand-Abravanel, J. Izopet, and L. Rostaing, "Hepatitis e virus: what transplant physicians should know," American Journal of Transplantation, vol. 12, no. 9, pp. 22812287, 2012.

[9] H. R. Dalton, R. Bendall, S. Ijaz, and M. Banks, "Hepatitis E: an emerging infection in developed countries," The Lancet Infectious Diseases, vol. 8, no. 11, pp. 698-709, 2008.

[10] B. Said, S. Ijaz, M. A. Chand, G. Kafatos, R. Tedder, and D. Morgan, "Hepatitis e virus in England and Wales: indigenous infection is associated with the consumption of processed pork products," Epidemiology and Infection, vol. 142, no. 7, pp. 14671475, 2014.

[11] H. R. Dalton, S. D. Pas, R. G. Madden, and A. A. van der Eijk, "Hepatitis E virus: current concepts and future perspectives," 
Current Infectious Disease Reports, vol. 16, no. 4, article 399, 2014.

[12] V. Moal, A. Motte, M. Kaba, R. Gerolami, Y. Berland, and P. Colson, "Hepatitis E virus serological testing in kidney transplant recipients with elevated liver enzymes in 2007-2011 in southeastern France," Diagnostic Microbiology and Infectious Disease, vol. 76, no. 1, pp. 116-118, 2013.

[13] S. Ijaz, A. J. Vyse, D. Morgan, R. G. Pebody, R. S. Tedder, and D. Brown, "Indigenous hepatitis $\mathrm{E}$ virus infection in England: more common than it seems," Journal of Clinical Virology, vol. 44, no. 4, pp. 272-276, 2009.

[14] H. R. Dalton, W. Stableforth, P. Thurairajah et al., "Autochthonous hepatitis e in Southwest England: natural history, complications and seasonal variation, and hepatitis e virus IgG seroprevalence in blood donors, the elderly and patients with chronic liver disease," European Journal of Gastroenterology and Hepatology, vol. 20, no. 8, pp. 784-790, 2008.

[15] H. Dalton and E. Hepatitis, "The "new kid on the block" or an old friend?" Transfusion Medicine and Hemotherapy, vol. 41, no. 1, pp. 6-9, 2014.

[16] T. Hering, A. M. Passos, R. M. Perez et al., "Past and current hepatitis E virus infection in renal transplant patients," Journal of Medical Virology, vol. 86, no. 6, pp. 948-953, 2014. 


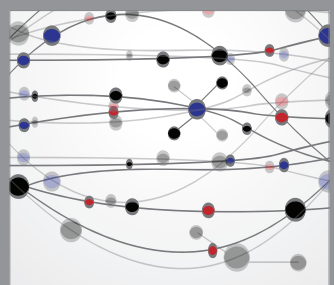

The Scientific World Journal
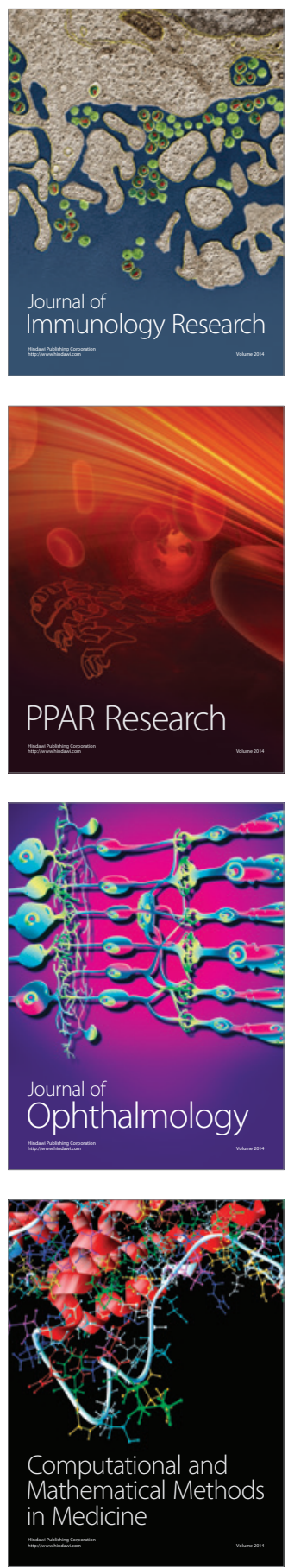

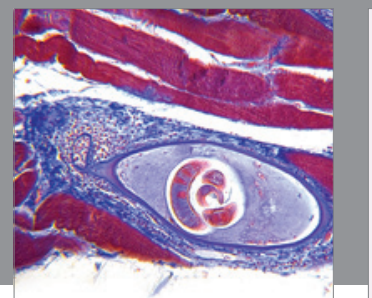

Gastroenterology

Research and Practice
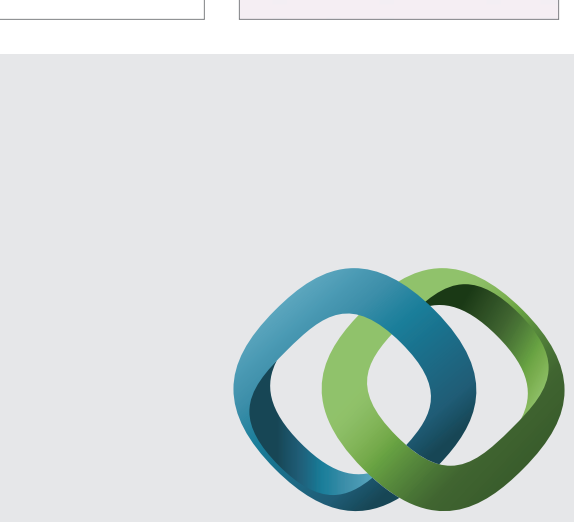

\section{Hindawi}

Submit your manuscripts at

http://www.hindawi.com
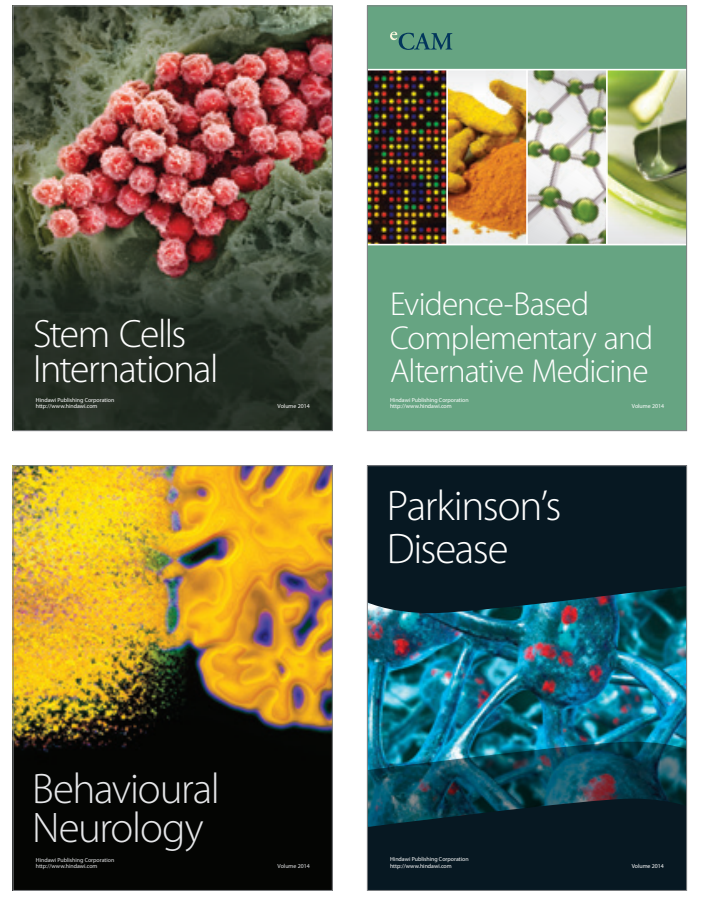
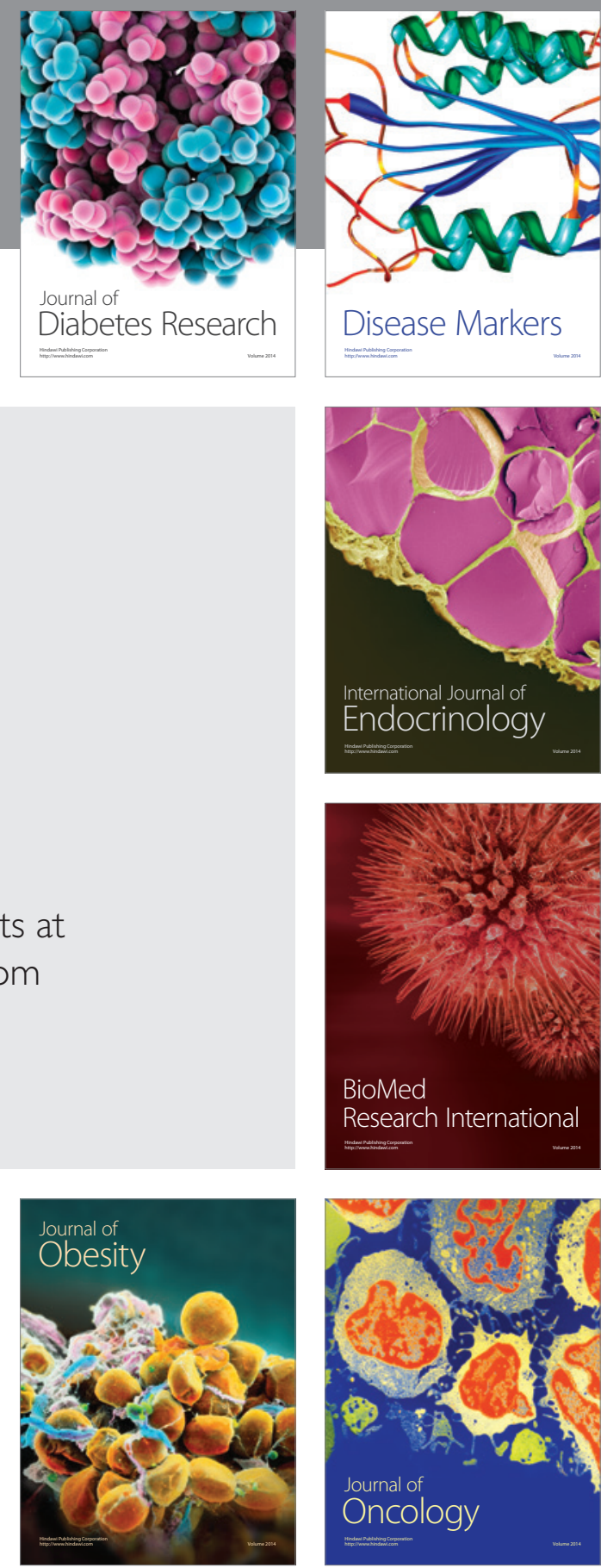

Disease Markers
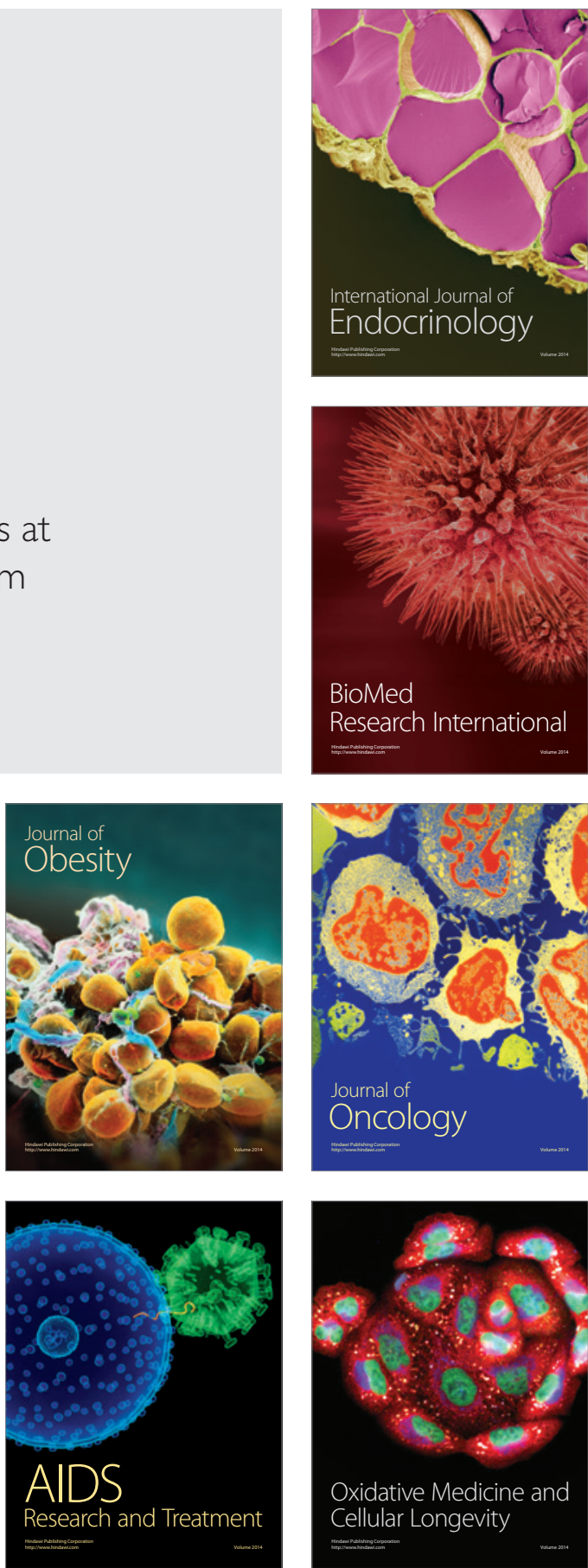